\title{
Capítulo 9
}

\section{Indicadores de vulnerabilidade e risco como subsídios à prevenção de impactos à sociobiodiversidade na bacia do rio Jari (AP-PA)/Brasil}

Alzira Marques Oliveira

Universidade Federal do Amapá

alzira.marques@unifap.br
Alan Cavalcanti da Cunha Universidade Federal do Amapá alancunha12@gmail.com

\section{Resumo}

Indicadores de risco e vulnerabilidade socioambiental são normalmente adotados pelo Programa de Risco de Desastre das Nações Unidas e frequentemente utilizados como referência mundial em análise de risco, subsidiando gestores públicos não somente na identificação de populações vulneráveis, mas também para potencializar ações e políticas ambientais de conservação da biodiversidade. Na presente investigação, o objetivo é estimar parâmetros estatísticos que quantifiquem o nível de risco socioambiental de duas comunidades urbanas localizadas no Baixo Rio Jari - AP/Brasil (Município de Laranjal do Jari-AP e distrito de Monte Dourado-PA). A metodologia apresenta as seguintes etapas: a) estimativa do nível do risco socioambiental, mensurado como o produto da ameaça (A) e da vulnerabilidade (V), ambas dependem de variáveis independentes socioeconômicas, sanitárias e ambientais; b) investigação de campo e aplicação de formulário contendo questões abertas e fechadas, para medir as 
variáveis independentes (socioambientais, climáticas, infraestrutura etc) in loco; c) análise de correlação múltipla não paramétrica (Spearman, p <0,05) - renda, escolaridade, localização de moradia, material utilizado na construção da casa, acesso ao abastecimento de água e redes de esgoto sanitário, entre outros). Essas variáveis foram utilizadas como explicativas de $\mathrm{V}$ e $\mathrm{R}$. Os resultados indicam variações do risco entre as duas localidades, com piores indicadores para Laranjal do Jari, em face de sua conjuntura histórica, infraestrutural e socioeconômica. A análise de cenários de risco estimados por $\mathrm{R}=\mathrm{A} \times \mathrm{V}$, para a cidade Laranjal do Jari e Monte Dourado, dependendo da condição climática (normal, seco ou chuvoso, muito seco ou muito chuvoso) variaram em até $300 \%$, de acordo com as respectivas áreas geográficas (por exemplo, bairros mais vulneráveis de Laranjal do Jari). Conclui-se que as populações com maior vulnerabilidade representam potencial e consequentemente maior grau de ameaça ao patrimônio socioambiental e à diversidade biológica, principalmente quando associadas à ocorrência de eventos climáticos extremos.

Palavras-chave: Cenários. Ameaças. Eventos Extremos. Variabilidade. Matriz de Correlação.

\subsection{Introdução}

A presente investigação tem como objetivo gerar indicadores de risco (R) e vulnerabilidade $(\mathrm{V})$ socioambiental a partir da quantificação de variáveis socioambientais (urbanas) e climáticas (eventos extremos) e suas associações concernentes a desastres naturais e ameaça à sociobiodiversidade local na bacia hidrográfica do rio Jari.

O baixo trecho da bacia do rio Jari é reconhecido como muito suscetível às variações hidroclimáticas e sanitárias, onde se tem observado sensível deteriorização da qualidade da água, potencialização do nível de eutrofização (ecossistemas aquáticos lóticos) e novas ameaças relacionadas à segurança de barragem Santo António do Jari (ABREU; CUNHA, 2016; OLIVEIRA; CUNHA, 2014).

Além da problemática local, os dados sobre a ocorrência de inundações e seus impactos no Brasil também são preocupantes. Por exemplo, as inundações bruscas, no período de 2000-2003, deixaram um prejuízo à nação de $\mathrm{R} \$ 176 \mathrm{mi}$ lhões e aproximadamente 6,5 mil desabrigados (MARCELINO, 2007). E somente no primeiro semestre de 2010 as enchentes atingiram seis estados brasileiros, como Pernambuco, onde 49 municípios têm sofrido com efeitos de temporais. Destes municípios, treze decretaram situação de emergência e nove, estado de calamidade. Mais de 14 mil pessoas ficaram desabrigadas. Em Alagoas, 53 mil 
pessoas ficaram desabrigadas naquele período, com falta de água potável e de alimento em algumas cidades (ALVES; 2015).

$\mathrm{Na}$ Amazônia, em 2004, as enchentes afetaram 1,2 milhões de pessoas. Apesar de se encontrarem sob constante ameaça de inundações, os municípios da região de várzea não têm um plano de emergência específico para enfrentar tais calamidades. Em 2009, mais de 180 mil pessoas foram atingidas pela ocorrência de chuvas no interior do Amazonas, com prejuízos de aproximadamente 6 milhões de reais (EM-DATA, 2016).

No estado do Amapá, estudos realizados por Oliveira e Cunha (2015) sugerem que as enchentes foram os eventos climáticos que mais têm causado prejuízos em termos econômicos e sociais, além de danos humanos e materiais, na última década. A primeira efetivamente registrada ocorreu em 2000, a segunda em 2006 e a terceira em 2008. Apenas a enchente de 2000 atingiu aproximadamente $70 \%$ do centro urbano da cidade, causando danos da ordem de 28,7 milhões, superior ao PIB do município de Laranjal do Jari-AP.

Em 2000, essas enchentes na bacia do rio Jari atingiram severamente o município de Laranjal do Jari e alguns de seus impactos foram considerados altamente significativos, tanto pela ausência de um sistema de previsão e monitoramento do tempo, clima e recursos hídricos efetivo quanto pela falta de planejamento preventivo para seu enfrentamento. Por exemplo, a vulnerabilidade socioambiental (ocupação em área de várzea), a falta de preparo da população para agir durante a ocorrência dos eventos, associadas à vulnerabilidade climática, são as condições mais favoráveis a esses desastres (LUCAS et al., 2010).

Em decorrência de potenciais mudanças e cenários ambientais em termos de vulnereabilidade $(\mathrm{V})$, tem sido também observada uma tendência de elevação dos riscos desses impactos difusos (ambientais, climáticos, hidrológicos, econômicos etc) em relação ao clima (hidrologia da bacia) bem como a deteriorização das condições urbanas locais.

Desse modo, a presente pesquisa é um diagnóstico simplificado elaborado para representar minimamente as condições econômicas e socioambientais do município de Laranjal do Jari-AP e do distrito de Monte Dourado-PA, ambos localizados no baixo curso da bacia hidrográfica do rio Jari (região de maior risco socioambiental). Apesar do foco ser as zonas urbanas, considera-se toda a dimensão da unidade territorial integrada da bacia, inclusive a influência de unidades de conservação e seus conflitos com zonas urbanas (OLIVEIRA; CUNHA, 2015).

Com esse foco, pretende-se mapear os locais (bairros, cidade etc) onde as populações estão mais vulneráveis e entender quais seriam as variáveis que melhor explicam essas condições de risco $(\mathrm{R})$ e vulnerabilidade $(\mathrm{V})$ socioambientais locais utilizando-se de uma análise de risco. Com efeito, espera-se oferecer uma medida quantitativa de informação para subsidiar a elaboração de políticas públicas do 
setor de Defesa Civil em associação com tomadas de decisão em gestão da conservação da biodiversidade.

\subsection{Referencial teórico}

\subsubsection{Risco e vulnerabilidade}

$\mathrm{Na}$ literatura internacional, risco é a probabilidade de ocorrerem consequências danosas ou perdas esperadas (mortos, feridos, edificações destruídas e danificadas etc) como resultado de interações entre um perigo natural e as condições de vulnerabilidade local (UNDP, 2004). Em nível de Defesa Civil brasileira, o risco é a probabilidade de ocorrência de um acidente ou evento adverso, relacionado com a intensidade dos danos ou perdas resultantes deles (BRASIL, 2007).

Veyret (2007) define "risco" como uma situação relacionada à percepção de uma possível catástrofe, a qual uma população ou um indivíduo percebe e de que pode sofrer seus efeitos. Zanirato et al (2008) afirmam que o "risco não é algo apenas a ser medido, mas pode ser apreendido e qualificado na perspectiva da sociedade do medo e do risco, também definido como um evento cultural que remete para além da condição de indivíduo".

Em geral, a ocorrência do risco pode ser observada através da associação de duas variáveis: a vulnerabilidade da população $(\mathrm{V})$, como um processo socialmente construído, com a suscetibilidade dos lugares como parte de uma dinâmica planetária (VEYRET, 2007; CUTTER et al., 2003).

O risco (R) é componente importante da subdivisão da análise de perigo e da análise do risco para estudo de perigos naturais. O nível do risco varia dependendo de três fatores: perigo, exposição e vulnerabilidade (MARANDOLA; HOGAN, 2004).

Tobin e Montz (1997) definem o risco como resultado de uma probabilidade de ocorrência de eventos particulares e de previsão de perda, que deve ser avaliado de acordo com as tendências históricas. Essa informação propiciada pela tendência histórica é importante para avaliar o risco técnico, ainda que não traduza ou indique o número de exposição de um perigo ou as perdas esperadas por um evento específico.

A Defesa Civil brasileira (Brasil, 2007) a expressão que melhor define risco é:

$\mathrm{R}=\mathrm{A} \times \mathrm{V}$

Onde:

$\mathrm{R}=\mathrm{Risco}$

$\mathrm{A}=$ Ameaça

$\mathrm{V}=$ Vulnerabilidade 
Essa relação serve para explicar a interação com a magnitude do evento ou acidente, definindo os efeitos adversos medidos em termos de intensidade dos danos previstos. Por exemplo, com a crescente importância dos desastres, tem-se discutido a questão de "vulnerabilidade (V)", a qual passa a ser tema atual. De modo geral, ela pode ser definida como a probabilidade de uma comunidade, exposta a uma ameaça natural, segundo um grau de fragilidade de elementos (infraestrutura, moradia, atividades produtivas, grau de organização, sistemas de monitoramento e alerta, desenvolvimento político institucional, entre outros), sofrer danos humanos e materiais. A magnitude desses danos, por sua vez, está relacionada com o grau de vulnerabilidade (MOSER, 1998; MASKREY, 1989; DESCHAMPS, 2007; CARDONA, 1994).

A vulnerabilidade $(\mathrm{V})$ pode ser analisada de diferentes pontos de vista (físico, social, político, tecnológico, ideológico, cultural e educativa, ambiental, institucional), mesmo que todos eles, de alguma maneira, estejam relacionados à realidade atual da região. Sua gestão está associada diretamente com fatores de ordem antrópica, isto é, com a interação humana com a natureza. (CARDONA et al., 2005; MASKREY, 1989).

Segundo Salgado (2005), a vulnerabilidade e o risco estão associados às decisões de políticas que uma sociedade tem adaptado ao longo do tempo e depende, portanto, do desenvolvimento de cada região ou localidade. Para esse autor, o risco se origina como um produto da função que relaciona a priori a ameaça e a vulnerabilidade, é considerado intrínseco e latente dentro de uma sociedade, em função de seu nível, grau de percepção e meios para enfrentá-lo e depende das diretrizes marcadas pela mesma sociedade.

\subsubsection{Indicadores socioambientais}

Atualmente os problemas ambientais necessitam ser discutidos pelo poder público e pela sociedade, com o objetivo de gerar, adotar e implementar medidas mitigadoras e ações eficientes para minimizar os riscos por meio de medidas de controle e monitoramento.

Nesse sentido, os indicadores são elementos importantes para monitorar o progresso nas distintas dimensões, funcionando como ferramentas de apoio aos tomadores de decisões e àqueles responsáveis pela elaboração de políticas em todos os níveis, visto que os indicadores são parâmetros na avaliação de impactos sociais, econômicos e ambientais decorrentes de determinada atividade (GARCIA; GUERRERO, 2006).

Os indicadores são variáveis que possuem significados derivados de uma configuração científica, que refletem de forma sintética, no caso da vertente so- 
cioambiental, um interesse social pelo ambiente e que podem subsidiar processos de tomada de decisão (SOUZA et al., 2013).

Salvalagio, Lima e Schneider (2005) afirmam que indicadores quantificam e agregam dados que podem ser medidos e monitorados para determinar se está em curso uma mudança. Eles quantificam e simplificam fenômenos ajudando a compreender realidades complexas, mostrando as mudanças ocorridas em um sistema, havendo uma interligação entre indicadores, isto é, indicadores econômicos, ambientais, políticos, sociais e culturais.

$\mathrm{Na}$ visão de Chevalier et al. (1992), indicadores são variáveis que estão relacionadas com outras variáveis estudadas, que não podem ser diretamente observadas. Os indicadores são representações de determinados atributos de um sistema, cujo objetivo é agregar e quantificar informações de modo que sua significância fique mais aparente, simplificando as informações sobre fenômenos complexos e tentando melhorar, com isso, o processo de comunicação (VAN BELLEN, 2007).

Segundo Souza et al. (2013), no processo de elaboração de indicadores, devem ser utilizadas informações que de fato expressem a realidade das ações nas unidades, para que se possa analisar os fatores que estão contribuindo para a ocorrência de determinado fenômeno.

$\mathrm{Na}$ bacia do rio Jari, a construção de indicadores de vulnerabilidade e risco socioambiental é útil como base de criação de políticas públicas para atender às necessidades locais, uma vez que o monitoramento desses indicadores serve para formular políticas tanto sociais quanto ambientais.

\subsection{Materiais e métodos}

\subsubsection{Caracterização da área de estudo}

A bacia hidrográfica do rio Jari (Figura 9.1) está localizada na bacia hidrográfica contribuinte do rio Amazonas. Nela, o rio Jari se apresenta como um dos principais afluentes da margem esquerda ou calha norte da bacia amazônica. Com aproximadamente 845 quilômetros de extensão, o rio Jari nasce na Serra do Tumucumaque, em altitude da ordem de $656 \mathrm{~m}$, na fronteira com o Suriname. Sua foz na margem esquerda do rio Amazonas dista cerca de 300 quilômetros do Oceano Atlântico (FILIZOLA, 2005; EPE, 2010). 


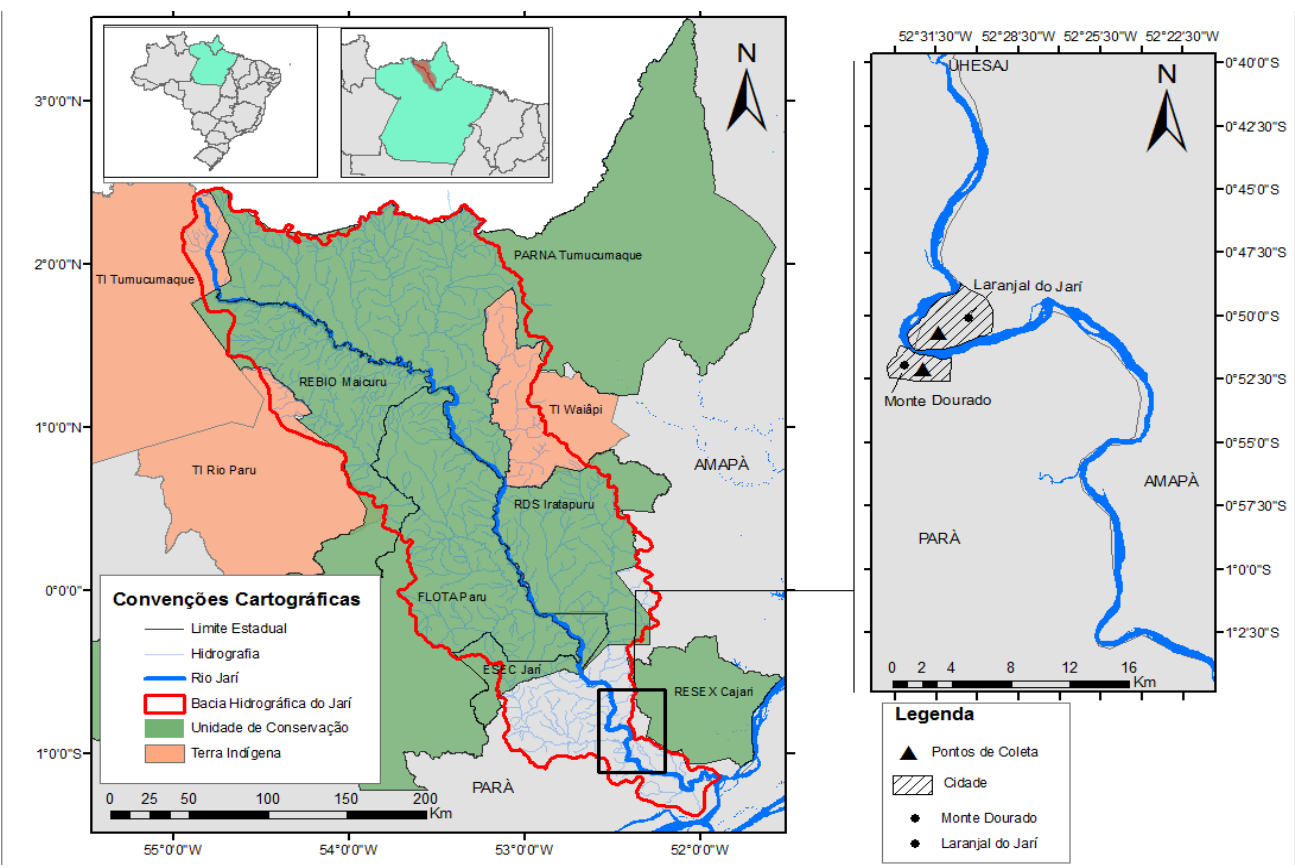

Figura 9.1 Área da bacia hidrográfica e as unidades de conservação inseridas no seu território. Fonte: adaptada de Hydros (2010).

A Figura 9.1 mostra a extensa biodiversidade existente na bacia do rio Jari. Essa bacia hidrográfica congrega uma vasta diversidade biológica e expressiva riqueza cultural. Esse patrimônio sociocultural é evidente na região, onde residem populações tradicionais, ribeirinhas e etnias indígenas, tais como: Waiãpi, Aparai, Wayana, Txutyana, Kaxuyana e Tiriyó. Na área da bacia hidrográfica do rio Jari, registram-se unidades de conservação de proteção integral, unidades de conservação de uso sustentável e terras indígenas, sendo que $77 \%$ são áreas de unidades de conservação e 10\% são terras indígenas (EPE, 2010; DIAS et al., 2016).

A elevação média da bacia é da ordem de $330 \mathrm{~m}$, variando de 20 a $30 \mathrm{~m}$, a montante da cachoeira de Santo Antônio, até 656 m nas cabeceiras, onde está localizado o Parque Nacional das Montanhas do Tumucumaque, região pouco conhecida e pouco explorada, de difícil acesso e ocupada tradicionalmente por populações ribeirinhas e indígenas (FILIZOLA, 2005).

Segundo a EcologyBrasil (2009), o rio Jari corre encaixado em vale aberto em "U", típico curso de drenagem escavado em embasamento sedimentar de climas equatoriais. Caudaloso durante todo ano, o rio Jari ainda não apresenta restrições associadas à indisponibilidade frente à demanda. A influência de maré em seu baixo curso garante a navegabilidade no rio o ano todo, independentemente das variações na vazão de montante (EPE, 2010; ABREU; 2014). 
Em grande parte de seu trecho o rio Jari serve de divisor entre o estado do Amapá e o do Pará, territórios de suas margens esquerda e direita, respectivamente. Ao longo da margem esquerda do rio Jari, está presente o município de Laranjal do Jari, exceto nos 80 quilômetros próximos na confluência com o Amazonas, onde localiza-se Vitória do Jari.

A população de Laranjal do Jari estimada em 2010 era de 39.942 habitantes. A área tem cerca de $30.971 \mathrm{~km}^{2}$, o que resulta em uma densidade demográfica média de 1,29 hab/km². Seus limites são Oiapoque, Pedra Branca do Amapari, Mazagão, Vitória do Jari e o estado do Pará, (cidade de Monte Dourado, no município de Almeirim), e ainda com Suriname e Guiana Francesa (IBGE, 2010).

$\mathrm{Na}$ margem direita, está localizado o distrito de Monte Dourado, onde se verifica considerável estado de alteração dos ecossistemas nativos, na maior parte associado ao uso para plantio florestal e exploração mineral pelo complexo industrial da Jari Celulose. Distinguem essa margem os elementos: a) extensa e evidente alteração da cobertura florestal nativa; b) mudança do uso do solo para atividade silvicultural, basicamente plantio de espécies florestais exóticas, como o eucalipto; c) intrincada malha viária rural voltada ao manejo florestal; d) desenvolvimento da economia industrial na localidade de Munguba-PA, com a presença de porto, mineração, termoelétrica e usina de celulose; e) planejamento urbano, ainda que concentrado em Monte Dourado, no distrito de Almeirim-PA (ECOLGYBRASIL; 2009; ABREU, 2014).

O parcelamento desenhado pelo rio Jari distingue, em certa medida, o quadro de conservação da paisagem e a ocupação da região. Enquanto na margem amapaense, município de Laranjal do Jari-AP, os ecossistemas terrestres se apresentam mais íntegros, na margem paraense, município de Almeirim, ocorre intenso manejo florestal para produção da matéria-prima celulose, além da remoção da vegetação nativa em largos trechos (ABREU, 2014).

\subsubsection{Elaboração de indicadores de vulnerabilidade e risco socioambiental}

Para a elaboração de indicadores de vulnerabilidade e de risco socioambiental dos munícipio de Laranjal do Jari-AP e Monte Dourado-PA foi considerado o nível de risco a partir da equação $\mathrm{R}=(\mathrm{A} \times \mathrm{V})$, onde $(\mathrm{R})$ é o risco, $(\mathrm{A})$ corresponde à ameaça climática à qual a bacia hidrográfica está exposta e (V) é o nível de vulnerabilidade local considerando variáveis socioeconômicas (renda, fonte de renda, escolaridade, abastecimento de água) e variáveis físico-ambientais (localização, material da casa, destino do lixo e esgotamento sanitário). Essas variáveis foram selecionadas com base em análise de correlação (LEVINE et al., 2005).

Segundo Lucas et. al. (2010) e Silveira (2014), a bacia do Jari apresenta três condições climáticas distintas, consideradas na pesquisa como a ameaça (A). A primeira é a de normalidade, a segunda é a de período seco ou chuvoso e o 
terceiro é a de período muito chuvoso ou muito seco. A partir desses dados, foram construídos três cenários de risco, de acordo com as ameaças e com a vulnerabilidade socioambiental de cada cidade, conforme Tabela 9.1.

Tabela 9.1 Classificação da vulnerabilidade e do risco socioambiental.

\begin{tabular}{|l|l|l|l|l|l|}
\hline \multicolumn{2}{|c|}{ Risco $($ A $\times$ V) } & \multicolumn{2}{c|}{ Ameaça (A) } & \multicolumn{2}{c|}{ Vulnerabilidade (V) } \\
\hline Grau & Classes & Fator climático & Atributo numérico & Grau & Atributo numérico \\
\hline Baixo & $1-3$ & Normalidade & 1 & Baixa & 1 \\
\hline Médio & $4-6$ & Seco ou chuvoso & 2 & Média & 2 \\
\hline Alto & $7-9$ & Muito seco / muito chuvoso & 3 & Alta & 3 \\
\hline
\end{tabular}

Fonte: adaptada de UNDP, 2004.

Segundo a UNDP (2004), para se estimar os níveis de risco, é importante estabelecer valores que constituirão um indicativo para desenhar e planificar ações de redução do risco. $\mathrm{Na}$ presente pesquisa, os valores adotados foram escalonados de 1 a 9 .

\subsubsection{Coleta de dados}

Os dados socioeconômicos foram coletados por meio de entrevista, com a utilização do instrumento formulário. Em Laranjal do Jari, foram aplicados 159 formulários em sete bairros da área de zona de várzea do município de Laranjal do Jari, por sorteio. São eles: Mirilândia, Samaúma, Malvina, Comercial, Três irmãos, Santarém e Central. Em Monte Dourado, foram aplicados 33 formulários nos bairros: Facel, intemediária e Staff.

\subsubsection{Coleta de variáveis socioeconômicas e ambientais e análise de dados}

As variáveis selecionadas para compor este estudo deveriam atender a dois pressupostos: o primeiro era implicar fatores de vulnerabilidade social e ambiental (ISDR, 2002); o segundo, apresentar significativa correlação (significância) por meio da análises estatísticas multivariadas (UNDP, 2004), conforme dispostas no Quadro 9.1.

Quadro 9.1 Variáveis coletadas por meio do formulário para a matriz de correlação.

V1. Cidade (1-Monte Dourado; 2- Laranjal do Jari;)

V2. Bairro (1-Central; 2- Santarém; 3- N. Esperança; 4Comercial; 5- Malvina; 6- Três Irmãos; 7- Samauma; 8- Facel; 9- Intermediária; 10-Staff)
V3. Sexo (1- M; 2- F)

V4. Idade/anos

V5. Naturalidade (1- Pará; 2- Piauí; 3-Ceará; 4- Maranhão; 5- Amapá; 6- Outros estados) 
Quadro 9.1 Variáveis coletadas por meio do formulário para a matriz de correlação. (continuação)

V6. Razão da migração (1- Trabalhar; 2- Buscar emprego; 3- Outros)

\section{V7. Renda (R\$)}

V8 Fonte de renda (1-Vínculo empregatíicio; 2- Conta própria; 3-Aposentadoria)

V9. Escolaridade (1- Nenhuma; 2-Alfabetizado; 3- Ens. Fund. incompleto; 4- Ens. Fund. completo; 5- Ens. Médio incompleto; 6-Ens. Médio completo; 7- Ens. Superior incompleto; 8- Ens. Superior completo

V10. Número de pessoas da residência

V14. Condição do logradouro do domićlio (1- Asfalto; 2- Terra; 3- Ponte/passarela)
V15. Material da casa (1- Madeira; 2- Alvenaria; 3- Mista)

V16. Número de cômodos

V17. Banheiro (1-Interno; 2- Externo)

V18. Fornecimento de energia (1-Sim; 2- Não)

V19. Abastecimento de água (1-Rede geral com canalização interna; 2- Rede geral sem canalização interna; 3- Não há (pega no vizinho ou em outro bairro)

V20. Nota para o fornecimento de água

V21. Destino do lixo (1-Coleta da prefeitura ou particular; 2-Descarte; 3-Queima)

V22. Esgotamento sanitário (1- Rede de esgoto geral; 2- Céu aberto; 3- Direto no rio)

A análise foi realizada a partir dos parâmetros estatísticos, como o nível de explicabilidade $\left(\mathrm{R}^{2}\right)$ (ou correlação - ) das variáveis que caracterizam os fatores de vulnerabilidade. Estes foram avaliados segundo seus valores de vulnerabilidades socioeconômicas e ambientais. Esses fatores foram baseados nas variáveis cuja correlação foi significativa em relação à vulnerabilidade socioambiental, sendo escalonados entre 1 e 3, em que o valor mínimo é 1 e o valor máximo é 3, conforme Tabela 9.1. As variáveis socioeconômicas utilizadas foram: renda, fonte de renda, escolaridade, abastecimento de água. As variáveis físico-ambientais foram: localização, material da casa, destino do lixo e esgotamento sanitário, também utilizadas como indicadores socioambientais. Elas foram selecionadas a partir de análise de correlação entre elas.

Tabela 9.2 Variáveis com grau significativo de correlação de acordo com nível de vulnerabilidade.

\begin{tabular}{|c|c|c|c|c|}
\hline \multirow[b]{2}{*}{ Indicador } & \multirow[b]{2}{*}{ Variável } & \multicolumn{3}{|c|}{ Vulnerabilidade } \\
\hline & & $\begin{array}{c}\text { Baixa } \\
\text { Atributo } \\
\text { numérico: } 1\end{array}$ & $\begin{array}{c}\text { Média } \\
\text { Atributo } \\
\text { numérico: } 2\end{array}$ & $\begin{array}{c}\text { Alta } \\
\text { Atributo } \\
\text { numérico: } 3\end{array}$ \\
\hline \multirow{4}{*}{ Socioeconômicos } & Renda & $<4 S M$ & $>1<3 S M$ & $>1 S M$ \\
\hline & Fonte de renda & $\begin{array}{l}\text { Vinculo empregatício } \\
\text { Comércio } \\
\text { Empresário }\end{array}$ & Aposentadoria & $\begin{array}{l}\text { Sem renda / } \\
\text { Conta } \\
\text { Própria }\end{array}$ \\
\hline & Escolaridade & $\begin{array}{l}\text { Ensino Superior incompleto } \\
\text { Ensino Superior completo }\end{array}$ & $\begin{array}{l}\text { Ensino Fundamental } \\
\text { completo a Ensino médio } \\
\text { completo }\end{array}$ & $\begin{array}{l}\text { Nenhum/alfabetizado/ } \\
\text { Ensino Fundamental } \\
\text { incompleto }\end{array}$ \\
\hline & $\begin{array}{l}\text { Abastecimento } \\
\text { de água }\end{array}$ & Rede geral com canalização & $\begin{array}{l}\text { Rede geral sem } \\
\text { canalização }\end{array}$ & $\begin{array}{l}\text { Sem acesso à água } \\
\text { potável }\end{array}$ \\
\hline
\end{tabular}


Tabela 9.2 Variáveis com grau significativo de correlação de acordo com nível de vulnerabilidade. (contunuação)

\begin{tabular}{|c|c|c|c|c|}
\hline \multirow[b]{2}{*}{ Indicador } & \multirow[b]{2}{*}{ Variável } & \multicolumn{3}{|c|}{ Vulnerabilidade } \\
\hline & & $\begin{array}{c}\text { Baixa } \\
\text { Atributo } \\
\text { numérico: } 1\end{array}$ & $\begin{array}{c}\text { Média } \\
\text { Atributo } \\
\text { numérico: } 2\end{array}$ & $\begin{array}{c}\text { Alta } \\
\text { Atributo } \\
\text { numérico: } 3\end{array}$ \\
\hline \multirow{4}{*}{ Físico-ambientais } & $\begin{array}{l}\text { Localização do } \\
\text { terreno }\end{array}$ & Asfalto & Terra & $\begin{array}{l}\text { Ponte/ } \\
\text { passarela }\end{array}$ \\
\hline & Material da casa & Alvenaria & Mista & Madeira \\
\hline & Destino do lixo & $\begin{array}{l}\text { Coleta pela } \\
\text { prefeitura/por particular }\end{array}$ & Queima & Descarte \\
\hline & $\begin{array}{l}\text { Esgotamento } \\
\text { sanitário }\end{array}$ & Rede de esgoto & Fossa & $\begin{array}{l}\text { Direto no } \\
\text { rio ou a céu } \\
\text { aberto }\end{array}$ \\
\hline
\end{tabular}

Legenda: $\mathrm{SM}=$ salário mínimo

Para utilização na equação do risco, a vulnerabilidade socioambiental foi calculada por meio de valores normalizados atribuídos a cada variável por morador, posteriormente agregados por bairros.

Para o cálculo da vulnerabilidade, foi utilizada a seguinte equação:

$\mathrm{V}=\sum$ (Atributo numérico $) / \mathrm{n}$

Onde:

$\mathrm{V}=$ Vulnerabilidade

Atributo numérico=Soma dos valores atribuídos a cada indicador (1-3)

$\mathrm{n}$ = Número de variáveis selecionadas após análise de correlação

O International Strategy for Disaster Redution (ISDR, 2002) define risco como a probabilidade de ocorrer danos às pessoas, a bens e a atividades econômicas, resultantes da interação entre perigos naturais e condições de vulnerabilidade de um sistema social. O Programa das Nações Unidas para o Desenvolvimento (UNDP, 2004) propõe um indicador de risco que utilize não apenas o número de mortos e feridos como representante da vulnerabilidade, mas também varáveis socioeconômicas e ambientais.

Nesse sentido, a pesquisa adotou, no rol de variáveis, os fatores socioeconômicos e ambientais, adotando a equação $\mathrm{R}=(\mathrm{A} \times \mathrm{V})$, visto que, nela, o risco congrega uma série de variáveis ambientais e socioeconômicas versus a vulnerabilidade. 


\subsection{Resultados e discussões}

$\mathrm{Na}$ presente pesquisa, um dos objetivos é disponibilizar ferramentas uteis à gestão pública, visando a diminuir ou a melhor identificar impactos causados por enchentes em áreas urbanas consideradas vulneráveis. Como resultado, oferecer a oportunidade de estabelecer parâmetros que auxiliem tanto na gestão de Defesa Civil quanto na elaboração de Políticas Públicas Ambientais, a fim de evitar potenciais impactos à biodiversidade bem como a garantir sua maior proteção.

\subsubsection{Indicadores de vulnerabilidade}

O levantamento socioeconômico e ambiental da população residente na área de várzea urbana de Laranjal do Jari compreende a área de risco resultante de ocupações irregulares, como afirma TUCCI (2002). A partir das informações de indicadores (socioeconômicos, físicos e ambientais) dessas áreas, foi elaborada uma escala de valoração da vulnerabilidade.

Por meio de uma matriz de correlação (Levine et al., 2005; Ayres et al., 2005), foram selecionadas somente as variáveis que atenderam aos pressupostos da pesquisa, correlação ( $\mathrm{R}$ de correlação) linear com $\mathrm{p}<0.0001$ (Tabela 9.3), isto é, alto grau de significância entre as variáveis.

As variáveis mostradas na Tabela 9.3 caracterizam os fatores de riscos e de vulnerabilidades nas cidades de Laranjal do Jari-AP e Monte Dourado. São eles: renda, fonte de renda, escolaridade, localização da residência, tipo de material da casa, abastecimento de água, coleta de lixo domiciliar e esgotamento sanitário.

Tabela 9.3 Correlação não paramétrica significativas das variáveis socioambientais (V).

\begin{tabular}{|c|c|c|c|c|c|c|c|}
\hline Variável & V8 & V9 & V14 & V15 & V19 & V21 & V22 \\
\hline V7 & $\begin{array}{l}r=0.5577 \\
R^{2}=0.3111 \\
p=<0.0001\end{array}$ & $\begin{array}{l}r=0.7974 \\
R^{2}=0.6358 \\
p=<0.0001\end{array}$ & $\begin{array}{l}r=0.7497 \\
R^{2}=0.5621 \\
P=<0.0001\end{array}$ & $\begin{array}{l}r=-0.3354 \\
R^{2}=0.1125 \\
p=<0.0001\end{array}$ & $\begin{array}{l}r=0.5650 \\
R^{2}=0.3192 \\
p=<0.0001\end{array}$ & $\begin{array}{l}r=0.4390 \\
R^{2}=0.1927 \\
p=<0.0001\end{array}$ & $\begin{array}{l}r=-0.7328 \\
R^{2}=0.5370 \\
p=<0.0001\end{array}$ \\
\hline V8 & - & $\begin{array}{l}r=0.7604 \\
R^{2}=0.5782 \\
p=<0.0001\end{array}$ & $\begin{array}{l}r=0.6899 \\
R^{2}=0.4759 \\
p=<0.0001\end{array}$ & $\begin{array}{l}r=0.4048 \\
R^{2}=0.1638 \\
p=<0.0001\end{array}$ & $\begin{array}{l}r=0.5495 \\
R^{2}=0.3019 \\
p=<0.0001\end{array}$ & $\begin{array}{l}r=0.5858 \\
R^{2}=0.3431 \\
p=<0.0001\end{array}$ & $\begin{array}{l}r=0.6480 \\
R^{2}=0.4198 \\
p=<0.0001\end{array}$ \\
\hline V9 & $\begin{array}{l}r=-0.7604 \\
R 2=0.5782 \\
p=<0.0001\end{array}$ & - & $\begin{array}{l}r=0.8570 \\
R^{2}=0.7344 \\
p=<0.0001\end{array}$ & $\begin{array}{l}r=0.4369 \\
R^{2}=0.1909 \\
p=<0.000\end{array}$ & $\begin{array}{l}r=0.6400 \\
R^{2}=0.4096 \\
p=<0.0001\end{array}$ & $\begin{array}{l}r=0.5686 \\
R^{2}=0.3228 \\
p=<0.0001\end{array}$ & $\begin{array}{l}r=-0.7758 \\
R^{2}=0.6018 \\
p=<0.0001\end{array}$ \\
\hline
\end{tabular}


Tabela 9.3 Correlação não paramétrica significativas das variáveis socioambientais (V). (continuação)

\begin{tabular}{|c|c|c|c|c|c|c|c|}
\hline Variável & V8 & V9 & V14 & V15 & V19 & V21 & V22 \\
\hline V14 & $\begin{array}{l}r=0.6899 \\
R^{2}=0.4759 \\
p=<0.0001\end{array}$ & $\begin{array}{l}r=-0.8570 \\
R^{2}=0.7344 \\
p=<0.0001\end{array}$ & - & $\begin{array}{l}r=0.6715 \\
R^{2}=0.4509 \\
p=<0.0001\end{array}$ & $\begin{array}{l}r=0.6944 \\
R^{2}=0.4822 \\
p=<0.0001\end{array}$ & $\begin{array}{l}r=0.5459 \\
R^{2}=0.2990 \\
p=<0.0001\end{array}$ & $\begin{array}{l}r=0.7757 \\
R^{2}=0.6017 \\
p=<0.0001\end{array}$ \\
\hline V15 & $\begin{array}{l}r=-0.4048 \\
R^{2}=0.1638 \\
p=<0.0001\end{array}$ & $\begin{array}{l}r=0.4369 \\
R^{2}=0.1909 \\
p=<0.0001\end{array}$ & $\begin{array}{l}r=0.6715 \\
R^{2}=0.4509 \\
p=<0.0001\end{array}$ & - & $\begin{array}{l}r==0.5015 \\
R^{2}=0.2515 \\
p=<0.0001\end{array}$ & $\begin{array}{l}r=0.3839 \\
R^{2}=0.1474 \\
p=<0.0001\end{array}$ & $\begin{array}{l}r=-0.3550 \\
R^{2}=0.1260 \\
p=<0.0001\end{array}$ \\
\hline V19 & $\begin{array}{l}r=0.5495 \\
R^{2}=0.3019 \\
p=<0.0001\end{array}$ & $\begin{array}{l}r=0.6400 \\
R^{2}=0.4096 \\
p=<0.0001\end{array}$ & $\begin{array}{l}r=0.6944 \\
R^{2}=0.4822 \\
p=<0.0001\end{array}$ & $\begin{array}{l}r=-0.5015 \\
R^{2}=0.2515 \\
p=<0.0001\end{array}$ & - & $\begin{array}{l}r=0.4617 \\
R^{2}=0.2132 \\
p=<0.000\end{array}$ & $\begin{array}{l}r=0.6349 \\
R^{2}=0.4031 \\
p=<0.0001\end{array}$ \\
\hline V21 & $\begin{array}{l}r=0.5858 \\
R^{2}=0.3431 \\
p=<0.0001\end{array}$ & $\begin{array}{l}r=0.5686 \\
R^{2}=0.3228 \\
p=<0.0001\end{array}$ & $\begin{array}{l}r=0.5459 \\
R^{2}=0.2990 \\
p=<0.0001\end{array}$ & $\begin{array}{l}r=-0.3839 \\
R^{2}=0.1474 \\
p=<0.0001\end{array}$ & $\begin{array}{l}r=0.4617 \\
R^{2}=0.2132 \\
p=<0.0001\end{array}$ & - & $\begin{array}{l}r=0.5072 \\
R^{2}=0.2573 \\
p=<0.0001\end{array}$ \\
\hline
\end{tabular}

$\mathrm{Na}$ linguagem coloquial, o termo "significante" quer dizer "algo importante" ao passo que, na linguagem estatística, esse termo tem o significado de "provavelmente verdadeiro" e, portanto, não resultante de uma situação aleatória. Um achado científico pode ser verdadeiro sem ser necessariamente importante. Quando os estatísticos informam que um resultado é "altamente significante", isso significa que a hipótese que está sendo testada é muito provavelmente verdadeira (LEVINE et al., 2005).

As populações mais vulneráveis e sujeitas aos impactos dos desastres naturais são as de baixa renda (PNUD, 2009), fato este corroborado pela presente análise para a população de várzea de Laranjal do Jari.

Detalhando a Tabela 9.3, o indicador "renda mensal" informa que $60 \%$ das famílias vivem com menos de um salário mínimo (SM) por mês e que $40 \%$ recebem entre um e dois SM, mostrando uma situação econômica caracterizada como abaixo da linha da pobreza, já que não existem condições de custear todas as necessidades com esse valor.

Contrapondo-se à realidade de Laranjal do Jari, em Monte Dourado todos os chefes de família entrevistados são funcionários da empresa Jari Celulose SA. Nesse sentido, o indicador "renda" sugere que as pessoas residentes nesse distrito desfrutam de boa qualidade de vida, pois ela permite acesso a serviços e produtos de qualidade. A renda da população entrevistada na cidade de Monte Dourado encontra-se na faixa de três a quatorze salários mínimos. 
Em relação à fonte de renda dos chefes de família das duas cidades, em Laranjal do Jari a situação do trabalho autônomo caracteriza uma situação de alta vulnerabilidade, já que, na ocorrência de enchentes, essas pessoas ficam totalmente fragilizadas, simplesmente porque toda a família depende dessa renda. Já em Monte Dourado, a realidade é inversa, pois todos os entrevistados têm fonte de renda segura, uma vez que são funcionários da empresa Jari Celulose ou de empresas terceirizadas.

Quanto ao indicador "escolaridade", verifica-se uma triste realidade para a população de Laranjal do Jari, embora o acordo firmado na Conferência Mundial para redução de desastre tivesse como meta atingir o primário universal, assegurando que, até 2015, a população de todo o mundo, especialmente as crianças, tivessem que concluir um ciclo completo de ensino primário (UN, 2005).

Considerando a taxa de escolaridade da população de Laranjal do Jari, nota-se que o índice de escolaridade é muito baixo. Constatou-se que pessoas sem nenhum grau de instrução equivalem a $10 \%$ dos entrevistados. Vinte e nove por cento afirmam saber escrever o próprio nome e ler algumas palavras. Trinta e quatro por cento possuem o Ensino Fundamental incompleto. Vinte e quatro por cento representam a soma das pessoas com Ensino Fundamental completo e Ensino Médio incompleto. Apenas três por cento dos entrevistados na área de estudo possuem Ensino Médio completo. Os dados sobre escolaridade mostram que, na realidade, os bairros da área de várzea de Laranjal do Jari não têm acompanhado o crescimento no nível educacional, contribuindo para uma realidade de vida cada vez mais degradada.

Em Monte Dourado, o indicador educacional aponta para alto índice de ensino. O nível mais baixo de escolaridade dos chefes de família nesse distrito é o Ensino Médio incompleto, o que corresponde a $6 \%$ dos entrevistados. A maioria dos chefes de família possui Ensino Superior completo.

Em Laranjal do Jari, o indicador "localização do terreno" aponta que $77 \%$ da população possui sua residência em pontes e passarelas, onde as condições de vida são precárias. Dezesseis por cento dos entrevistados residem em rua de terra. Isso se deve a morarem em uma das principais ruas de acesso à parte alta da cidade. Sete por cento residem em ruas de asfalto. Em Monte Dourado, a população é significativamente privilegiada, pois suas ruas são asfaltadas com alto padrão de qualidade. Percebe-se um imenso descompasso entre a população que desfruta dos benefícios do desenvolvimento econômico e as excluídas desse processo (PNUD, 2009; VEYRET, 2007).

O indicador do tipo de material que a casa é construída sugere que $79 \%$ dos domicílios em Laranjal do Jari foram construídos de madeira, material frágil na ocorrência de incêndios e enchentes. Vinte e um por cento das casas são mistas, isto é, parte de alvenaria e parte de madeira. Em Monte Dourado, todas as casas são de alvenaria. Possuem área ampla e congregam toda uma infraestrutura ne- 
cessária para qualidade de vida urbana, como água tratada e rede geral de esgoto, rede de drenagem e coleta de lixo.

Laranjal do Jari apresenta uma situação precária quanto o acesso a agua potável fornecida pela rede pública. Apenas $21 \%$ dos entrevistados afirmam receber em suas residências esse serviço. Quarenta por cento da população recebe água fora de casa em instalações improvisadas. Trinta e nove por cento não têm acesso nem dentro e nem fora de casa. Para essas pessoas, resta a alternativa de buscar água para consumo em bairros próximos.

Em relação às condições de saneamento, em Laranjal do Jari, observa-se que a prefeitura promove a coleta de lixo e $44 \%$ dos entrevistados afirmam que têm acesso a esse serviço público. Entretanto, mesmo havendo coleta de lixo, 51\% dos moradores descartam seus resíduos direto na várzea e $5 \%$ dos moradores afirmam queimar o lixo domiciliar.

Uma das consequências do despejo de lixo a céu aberto é o assoreamento, que consiste na acumulação de partículas sólidas (sedimento) em meio aquoso. As altas concentrações de sedimentos em canal fluvial podem provocar o aumento da turbidez, a redução na penetração de luz, a diminuição da fotossíntese com consequente morte de peixes pela falta de oxigênio, entre outros problemas (OLIVEIRA, 2014).

Em Laranjal do Jari, constata-se a ausência de esgotamento sanitário. A população lança seus dejetos direto do rio $(38 \%)$ ou direto nas áreas de várzea a céu aberto (62\%). De modo inverso, no distrito de Monte Dourado, toda a população é atendida pela rede geral de esgoto.

A falta de esgotamento sanitário em Laranjal do Jari é um indicador de alto risco à população e ao meio ambiente. A situação de exposição de resíduos e de contaminação da água é um problema de saúde pública, pois várias doenças podem se disseminar na área, tais como cólera, diarreia, verminose, teníase etc. A disposição de esgoto de forma inadequada também é propícia para proliferação de insetos, mosquito, roedores e outros vetores de doenças.

No município de Laranjal do Jari, a vulnerabilidade socioambiental é consequência das condições socioeconômicas da população, da falta de planejamento urbano, do ordenamento (zoneamento), da ocupação do espaço e dos baixos investimentos em políticas públicas de saúde, educação, saneamento básico etc, o que aumenta consideravelmente a fragilidade da comunidade quando exposta a desastres naturais.

\subsubsection{Indicadores de riscos socioambientais}

Para análise dos resultados dos níveis de risco, foi considerada a escala da Tabela 9.1, resultando em três cenários básicos. Os cenários de ameaça têm como referência os fatores climáticos da região. 
No primeiro cenário (Tabela 9.4) de normalidade climática, verifica-se que o risco para todos os bairros da área estudada de Laranjal do Jari é baixo. Contudo, no segundo cenário, com presença de eventos de seca ou de chuva, o risco se eleva para o nível considerado médio. No último cenário, muito seco ou muito chuvoso, o risco torna-se alto.

Tabela 9.4 Cenários de ameaças climáticas e risco para a cidade Laranjal do Jari e Monte Dourado e Laranjal do Jari.

\begin{tabular}{|c|c|c|c|c|c|}
\hline & & $\begin{array}{l}\text { Grau de } \\
\text { vulnera- }\end{array}$ & & $\begin{array}{c}\text { (R) } \\
\text { isco }(A) \times\end{array}$ & \\
\hline Cida & /Bairro & socioam- & & Muito & \\
\hline & Marilândia & 2,70 & 2,70 & 5,40 & 8,10 \\
\hline & Sumaúma & 2,81 & 2,81 & 5,62 & 8,43 \\
\hline & Malvina & 2,78 & 2,78 & 5,56 & 8,34 \\
\hline $\begin{array}{l}\text { Laranial do } \\
\text { Jari }\end{array}$ & Comercial & 2,59 & 2,59 & 5,18 & 7,77 \\
\hline & Três Irmãos & 2,78 & 2,78 & 5,56 & 8,34 \\
\hline & Santarém & 2,60 & 2,60 & 5,20 & 7,80 \\
\hline & Central & 2,54 & 2,54 & 5,20 & 7,80 \\
\hline & Facel & 1,08 & 1,08 & 2,16 & 3,24 \\
\hline $\begin{array}{l}\text { Monte } \\
\text { Dourado }\end{array}$ & Intermediária & 1,05 & 1,05 & 2,10 & 3,15 \\
\hline & Staff & 1,00 & 1,00 & 2,00 & 3,00 \\
\hline
\end{tabular}

A zona urbana de Laranjal do Jari apresenta homogeneidade quanto às variáveis que caracterizam a vulnerabilidade socioambiental. Há necessidade de destacar que os bairros mais próximos do rio Jari são os que apresentam os maiores índices de risco, influenciados, consequentemente, pelos altos índices de vulnerabilidade socioambiental. Em todos os cenários de ameaça, o distrito de Monte Dourado é o que apresenta risco mais baixo. 
Como a vulnerabilidade socioambiental é muito baixa, o risco é o menor da escala observada (Tabela 9.4). Isso se deve a vários fatores, destacadamente qualidade de vida em decorrência do acesso a renda, serviços básicos de saúde, água potável etc. Entretanto, em período de chuva intensa, quando ocorrem as enchentes no município de Laranjal do Jari, os entrevistados (63\%) afirmaram que há incidência de assaltos e arrombamento em residências. Isso permite concluir que, embora de forma indireta, $86 \%$ da população que habita um local seguro sente os impactos causados pelos desastres naturais. Além disso, ocorrem impactos indiretos nas áreas de saúde, infraestrutura e segura pública.

\subsection{Conclusões}

A pesquisa teve como objetivo estimar de modo simplificado parâmetros estatísticos para mensurar o nível de risco socioambiental de duas comunidades urbanas localizadas no Baixo Rio Jari-AP/Brasil (município de Laranjal do Jari-AP e distrito de Monte Dourado-PA).

1) O município de Laranjal do Jari, por ser o mais atingido por enchentes em todo o estado do Amapá quando ocorrem eventos extremos de precipitação, tem sofrido as principais consequências dos riscos socioambientais, motivo de preocupação dos órgãos públicos de Defesa Civil e de infraestrutura devido às consequências negativas à população, considerada como eminentemente vulnerável e ao meio ambiente.

2) A condição de vulnerabilidade e risco é decorrente da situação econômica e de profunda exclusão social resultante do histórico de ocupação desordenada do Vale do Jari.

3) As variáveis socioeconômicas (renda, fonte de renda, escolaridade, localização da residência, tipo de material da casa, abastecimento de água) e físico -ambientais (coleta de lixo domiciliar e esgotamento sanitário) confirmaram a hipótese e são efetivamente os principais indicadores que explicam a variação de risco e a vulnerabilidade socioambiental.

4) O tema é relevante na medida em que futuros investimentos econômicos e financeiros, como os provenientes do setor de Defesa Civil e da Conservação da Biodiversidade, forneçam os elementos básicos das políticas ambientais para a conservação da biodiversidade.

\subsection{Agradecimentos}

Os autores agradecem ao Projeto Rede de Gestão Integrada de Monitoramento da Dinâmica Hidroclimática e Ambiental da Bacia do Jarí - estado do Amapá 
(Convênio 702813-2008-SUDAM-IEPA-UNIFAP) e CNPQ 303715/2015-4 pelo auxílio financeiro à pesquisa.

\subsection{Referências}

ABREU, C. H. M.; CUNHA, A. C. Qualidade da Água em Ecossistemas Aquáticos Tropicais Sob Impactos Ambientais no Baixo Rio Jari-AP: Revisão Descritiva. Biota Amazônia, v. 5, p. 119-131, 2015.

ABREU, C. H. M.; CUNHA, A. C. Uso de parâmetros físicos, químicos, microbiológicos e indicadores de estado trófico (IET) para avaliar qualidade da água em ecossistema tropical sob impactos ambientais. Dissertação (Mestrado em Biodiversidade Tropical)-Universidade Federal do Amapá, Macapá, 2016.

ABREU. C. H. M. Variações espaço-sazonais da qualidade da água e da hidrodinâmica em ecossistemas aquáticos sob impactos ambientais no baixo rio Jari-AP. 2014. Dissertação (Mestrado em Biodiversidade Tropical)-Universidade Federal do Amapá, Macapá, 2014.

ALVES, H. R. A gestão de riscos de desastres naturais no Brasil face às mudanças sociais e ambientais desencadeadas pelo processo de urbanização. 2015. Dissertação (Mestrado). Escola Superior Dom Helder Câmara, Belo Horizonte, 2015.

AYRES, M., AYRES JR., M., AYRES, D.L. \& SANTOS, A.S. BioEstat 5.0: Aplicações estatísticas nas áreas das ciências biológicas e médicas. Belém: Sociedade Civil Mamirauá, CNPq, 2005.

BRASIL (2007). Conferência Geral Sobre Desastres: para prefeitos, dirigente de instituições públicas e privadas e líderes comunitários. Ministério da Integração Nacional: Brasília, 2007.

CARDONA O. D. The need for rethinking the concepts of vulnerability and risk from a holistic perspective: a necessary review and criticism for effective risk management. In Bankoff, G., Frerks, G, and Hilhorst D. (Ed.) Mapping Vulnerability: Disasters, Development and People. London: Earthscan, 2003. Disponível em: <http://www.desenredando.org/public/articulos/index.html>. Acesso em: 20 ago. 2010 
et al. System of indicators for disaster risk management: main technical report. Manizales - Washington: Instituto de Estudios Ambientales Universidad Nacional de Colombia / Inter-American Development Bank, 2005.

CHEVALIER, S.; CHOINIERE, R.; BERNIER, L. et al. User guide to 40 Community Health Indicators. Community Health Division, Health and Welfare. Canada, Ottawa: 1992.

CUTTER, S. L.; BORUFF, B. J. ; SHIRLEY, W. L. Social vulnerability to environmental hazards. Social Science Quarterly, v. 84, n. 1, p. 242-261, 2003. demografia. Trabalho apresentado no XIV Encontro Nacional de Estudos populacionais. Caxambú-MG, 20-24 de Setembro de 2004.

DESCHAMPS, M. V. Vulnerabilidade socioambiental: o caso da região metropolitana de Curitiba. Seminário Inflexões urbanos - PPGTU PUCPR, 2007.

DIAS, T. C. A. C.; CUNHA, A. C.; Silva, J. M. C. Return on investment of the ecological infrastructure in a new forest frontier in Brazilian Amazonia. Biological Conservation, v. 194, p. 184-193, 2016.

ECOLOGYBRASIL. Estudo de Impacto Ambiental Usina Hidroelétrica Santo Antonio do Jari. Pará e Amapá, 2009.

EM-DAT. INTERNATIONAL DISASTER DATABASE. Center for Research on the Epidemiology of Disasters - CRED. Disponível em: Acesso em: mar. 2016

EPE - Empresa de Pesquisa Energética. Estudo de Inventário Hidroelétrico da Bacia Hidrográfica do rio Jari-AP e PA, 2010.

GARCIA, S.; GUERRERO, M. Indicadores de sustentabilidad ambiental en la gestión de espacios verdes: Parque urbano Monte Calvário, Tandil, Argentina. Rev. Geogr. Norte Gd., n. 35, p. 45-57, 2006.

IBGE (2010). Censo demográfico 2010. Disponível em: <www.ibge.gov.br>. Acesso em: 20 ago. 2016.

ISDR (2002). INTERNATIONAL STRATEGY FOR DISASTER REDUCTION. Living with risk: a global review of disaster reduction initiatives. Preliminary version. Geneva, Switzerland: UN/ISDR, 2002. Disponível em: <http://www. unisdr.org/we/inform/publications/8552>. Acesso em 27 ago. de 2016. 
LEVINE, D. M. et al. Estatística, Teoria e Aplicações. Maringá: Editora LTC, 2005.

LUCAS, E.W.M., BARRETO, N. J. C., CUNHA, A. C. Variabilidade hidrológica da Bacia do Rio Jari (AP): Estudo de caso do ano 2000. In: CUNHA., A.C.; SOUZA, E. B.; CUNHA, H. A. C. (Org.). Tempo, Clima e Recursos Hídricos: resultados do Projeto REMETAP no Amapá: Macapá: IEPA, 2010.

MARANDOLA JR., E. \& HOGAN, D. J. Vulnerabilidades e riscos: entre geografia e demografia. Trabalho apresentado no XIV Encontro Nacional de Estudos Populacionais. Caxambú-MG, 20-24 de Setembro de 2004.

MARCELINO, E. V. Desastres Naturais e Geotecnologias: conceitos básicos. Instituto Nacional de Pesquisas Espaciais. INPE Santa Maria, 2007. Disponível em: <http://www.inpe.br/crs/geodesastres/imagens/publicacoes/conceitosbasicos.pdf>. Acesso em: 10 ago. 2016

MASKREY, A. Disaster Mitigation: A Community Based Approach. Development Guidelines No.3 Oxford: Oxfam, 1989.

Disaster Mitigation: A Community Based Approach. Development Guidelines No.3 Oxford: Oxfam, 1989.

MOSER, C. The asset vulnerability framework: reassessing urban poverty reduction strategies. World Development, New York, v.26, n.1, 1998.

OLIVEIRA, A. M.; CUNHA, A. C. Análise de risco como medida preventiva de inundações na Amazônia: estudo de caso de enchente de 2000 em Laranjal do Jari-AP, Brasil. Ciência e Natura, v. 37, p. 110-118, 2015.

OLIVEIRA, B. S ; CUNHA, A. C. Correlação entre qualidade da água e variabilidade da precipitação no sul do Estado do Amapá. Revista Ambiente \& Água, v. 9, p. 261-275, 2014.

PNUD BRASIL (2009). Enchentes na Amazônia afetam 1,2 milhão. Disponível em: <http://www.pnud.org.br/meio_ambiente/reportagens/index. php?id01=650\&lay=ma>. Acesso em: 28 ago. 2016.

SALGADO, M., G. Reflexões em torno do conceito do risco natural e da dimensão do risco. Porto: Faculdade de Letras / Universidade do Porto, 2005 
SALVAGAGIO, R., LIMA; SCHNEIDER, M.C. Os indicadores ambientais como parâmetros de melhorias da Qualidade de vida. Anais. $2^{\circ}$ Seminário Nacional Estado e Políticas Sociais no Brasil, 2005.

SILVEIRA, J. S. Aspectos Hidroclimatológicos da Bacia do Rio Jari no período de 1968 a 2012. 2014. Trabalho de Conclusão de Curso. Universidade Federal do Amapá, Macapá, 2014.

SOUZA et al. Diagnóstico e construção de indicadores socioambientais participativos: experiências de um Programa de Extensão. Revista Brasileira de Ciências Ambientais, n. 30, 2013.

TOBIN, G. A.; MONTZ, B. E.. Natural hazards: explanation and integration. New York, London: The Guilford Press, 1997.

TORNEL, F. C. G. Algunas cuestiones sobre Geografía de los Riesgos. Scripta Nova. Revista Electrónica de Geografía y Ciencias Sociales. 2001. Revista Online. Disponível em: <http://www.ub.es/geocrit/sn-10.htm>. Acesso em 10 ago. 2016.

TUCCI, C E. M. Água no Meio Urbano. In.: REBOUÇAS, A. C.; BRAGA, B.; TUNDISI,

J.G. (Orgs). Águas doces no Brasil: capital ecológico,uso e conservação. São Paulo: Escrituras Editora, 2002.

UN. World Conference on Disaster Reduction. Kobe: UNGA; jan. 2005.

UNDP (2004). United Nations Development Programme /Bureau of Crisis Prevention and Recovery. A Global Report Reducing Disaster Risk: A Challenge for Development. New York: UNDP Bureau for Crisis Prevention and Recovery, 2004.

VAN BELLEN, H.M. Indicadores de sustentabilidade: uma análise comparativa. Rio de Janeiro: FGV, 2007.

VEYRET, Y. Os riscos: o homem como agressor e vítima do meio ambiente. São Paulo: Contexto, 2007.

ZANIRATO, S.H., RAMIRES, J.Z.S., AMICCI, A.G. N, RIBEIRO, Z.M., RIBEIRO, W.C. Sentidos do Risco: Interpretações teóricas. Revista Bibliográfica de Geografía e Ciencias sociales, v. 13, n. 785, 2008. 
